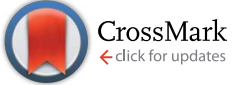

Cite this: Chem. Sci., 2016, 7, 2787

\title{
Facile syntheses of [3]-, [4]- and [6]catenanes templated by orthogonal supramolecular interactions $\uparrow$
}

\author{
Kai Wang, Chi-Chung Yee and Ho Yu Au-Yeung* \\ A water soluble [6]catenane consisting of two interlocking [3]catenanes was synthesised in $91 \%$ yield using \\ readily accessible precursors. The new strategy features the simultaneous use of orthogonal $\mathrm{Cu}^{+}-$ \\ phenanthroline and $\mathrm{CB}[6]-$ ammonium interactions for preorganising the precursors and the efficient $\mathrm{CB}$ \\ [6]-catalysed azide-alkyne cycloaddition as bond forming reactions for ring closing, resulting in high \\ structural complexity and fidelity of the products without compromising interlocking efficiency. A related \\ [4]catenane with three different types of macrocycles was also obtained in good yield.
}

Received 10th December 2015

Accepted 15th January 2016

DOI: $10.1039 / c 5 s c 04774 a$

www.rsc.org/chemicalscience

different numbers of complementary $\pi$-rich macrocycles, in which the formation of the [7]catenaned cage is favoured by shifting the equilibrium with a large excess of the $\pi$-rich macrocycle. ${ }^{6}$

Efficient strategies that are facile, general, controllable and applicable to $[n]$ catenanes that contain more interlocked macrocycles, different interlocking topology and ring connectivity are yet to be developed and will be necessary if the distinct properties of catenanes are to be developed into new molecular machines or incorporated into functional materials. ${ }^{1 a, b} \mathrm{We}$ anticipated that using more than one type of orthogonal supramolecular interaction as a template,${ }^{15}$ in conjunction with highly efficient bond forming reactions, could independently and simultaneously interlock multiple macrocycles without compromising the interlocking efficiency and simplicity in the precursor design. More importantly, products with fewer numbers of interlocking rings and other topological isomers can be minimised and therefore could give the desired $[n]$ catenanes in good yields. Here we describe a new strategy that catenane that has been isolated and characterised to date is a [7] catenane, with only a handful of $[n]$ catenanes $(n \geq 5)$ having been reported. ${ }^{1,5-14}$ The synthesis of these $[n]$ catenanes usually requires special reaction conditions and/or specific precursors and templates, and the yields are often modest. For example, the first [7]catenane reported by Stoddart and co-workers was assembled using $\pi$ donor-acceptor templation under an ultrahigh pressure of $12 \mathrm{kbar}$ in $27 \%$ yield, along with a series of related [4]-, [5]- and [6]catenanes. ${ }^{5}$ More recently, Nitschke and Sanders have reported an equilibrating system of tetrahedral metallocages with the six $\pi$-deficient edges interlocked with

Department of Chemistry, The University of Hong Kong, Pokfulam Road, Hong Kong, P. R. China.E-mail: hoyuay@hku.hk

$\dagger$ Electronic supplementary information (ESI) available: Synthetic procedures, NMR, MS, HPLC and UV-Vis data. See DOI: 10.1039/c5sc04774a

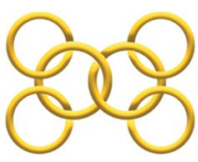

A

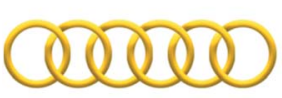

D

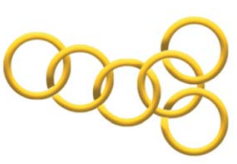

B

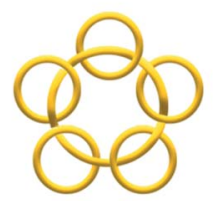

$\mathrm{E}$

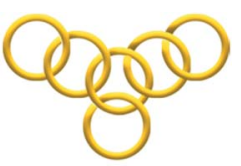

C

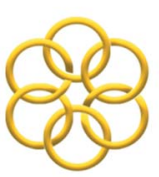

$\mathrm{F}$
Scheme 1 Some topoisomers of a [6]catenane with different ring connectivity: branched (A-C), linear (D), radial (E) and circular (F) [6] catenanes. In this work, only isomer A was obtained. 
combines $\mathrm{Cu}^{\mathrm{I}}$-phenanthroline coordination ${ }^{\mathbf{1 6}}$ and the iondipole and hydrophobic interactions between ammonium and cucurbit[6] uril $(\mathrm{CB}[6])^{17}$ with the $\mathrm{CB}[6]$-catalysed azide-alkyne cycloaddition $^{\mathbf{1 8}}$ as a ring-closing reaction to obtain a rare [6] catenane and a related [4]catenane with three different types of macrocycles in $91 \%$ and $84 \%$ yield, respectively.

\section{Results and discussion}

The [6]catenane C6, consists of two interlocking [3]catenanes, was synthesised through dropwise addition of $45 \mathrm{ml}$ of a $1 \mathrm{mM}$ solution of the diazide-CB[6] complex $\left[2 \subset \mathrm{CB}[6]_{2}\right]$ in $0.2 \mathrm{M}$ aq. $\mathrm{HCl}$ to an equal volume of a $0.5 \mathrm{mM}$ solution of the alkynefunctionalised $\mathrm{Cu}^{\mathrm{I}}$-phenanthroline complex $\left[\mathrm{Cu}(\mathbf{1})_{2}\right]\left[\mathrm{PF}_{6}\right]$ in DMF at $60^{\circ} \mathrm{C}$ over two hours, followed by stirring of the reaction mixture at the same temperature for two days (Scheme 2). LCMS analysis of the crude product mixture in solution revealed the formation of $\mathbf{C 6}$ in $91 \%$ yield along with $7 \%$ of the [3]catenane C3 as determined using the corresponding peak areas in the chromatogram. ${ }^{19}$ The latter can also be obtained in $85 \%$ yield from a similar reaction of $\mathbf{1}$ and $\mathbf{2}$ in the absence of $\mathrm{Cu}^{+}$(see the ESI $\dagger$ for details). Both C6 and C3 (and C4, vide infra) are water soluble in the form of chlorides, and their water solubility offers good opportunities for studying the unique mechanical motions of catenanes conferred by the aqueous environment which have not yet been extensively studied. ${ }^{20}$ Also, the branched structure of $\mathbf{C 6}$ represents a rare form of ring connectivity for high order $[n]$ catenanes when compared to the more common radial $[n]$ catenanes. ${ }^{7-13,21}$

ESI-MS analysis of $\mathbf{C 6}$ shows a series of peak clusters that are consistent with the molecular formulae of C6 in charge states of +5 to +8 (Fig. 1a). In addition, it is found that the peak at $\mathrm{m} / \mathrm{z}=$ 915.5, which corresponds to the +7 ion, has the strongest intensity, suggesting that the most abundant and stable form of the $\mathrm{Cu}^{+}$-coordinated [6] catenane under the ESI conditions is the one with six of the eight secondary amines being protonated. Nevertheless, under the acidic conditions used in the synthesis of C6, it is likely that all of the secondary amines are protonated and the ion-dipole interactions between the ammonium and $\mathrm{CB}[6]$ are maximised for the $\mathrm{CB}[6]$-catalysed click reactions. HRMS analysis of the peak at $m / z=915.5$ (the +7 ion) showed an isotopic pattern that is consistent with the expected molecular formula of the catenane (Fig. 1b). The interlocked structure of C6 was confirmed through $\mathrm{MS}^{2}$ and $\mathrm{MS}^{3}$ experiments. Fragmentation of the peak at $m / z=801.3$ resulted in fragments corresponding to $\mathbf{C} 3(\mathrm{~m} / \mathrm{z}=793.1)$ and its smaller fragments. Further fragmentation of the peak at $m / z=793.1$ produced a $\mathrm{MS}^{3}$ spectrum that is consistent with the $\mathrm{MS}^{2}$ spectrum of $\mathbf{C} 3$, supporting that $\mathbf{C 6}$ is composed of two interlocked $\mathbf{C 3}$ (Fig. 1c and d). It is noted from both the $\mathrm{MS}^{2}$ and $\mathrm{MS}^{3}$ spectra of $\mathbf{C 6}$ (and $\mathrm{MS}^{2}$ of $\mathbf{C 3}$, Fig. S39†) that the binding of $\mathrm{CB}[6]$ to the ammonium is so strong that the pseudorotaxane fragments are stable enough to be observed under the $\mathrm{MS}^{n}$ conditions. ${ }^{22}$

A sample of $\mathbf{C 6}$ purified through preparative HPLC was further characterised using NMR spectroscopy $\left({ }^{1} \mathrm{H},{ }^{13} \mathrm{C}\left\{{ }^{1} \mathrm{H}\right\}\right.$,

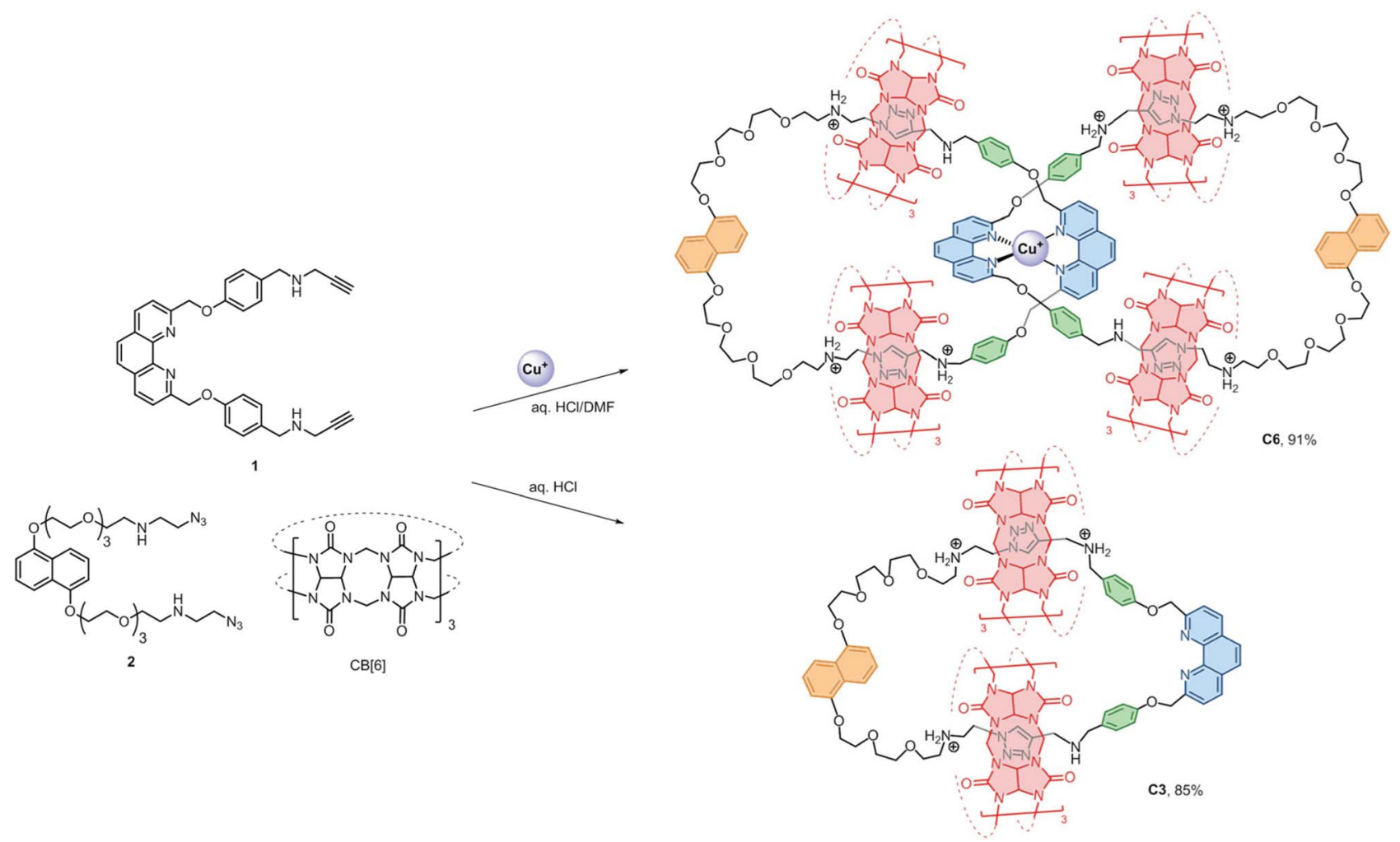

Scheme 2 Syntheses of C3 and C6. Structures of C6 and C3 are shown as the +7 and +3 ions which are the most abundant and stable forms of the catenanes as observed in the ESI-MS studies. Other protonation states of the catenanes are also observed in the MS studies. 

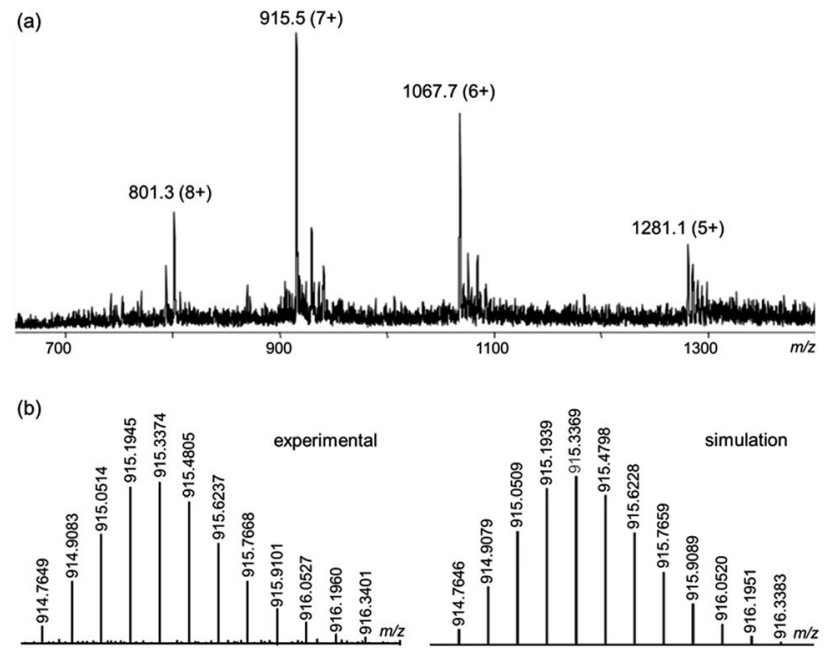

(c)
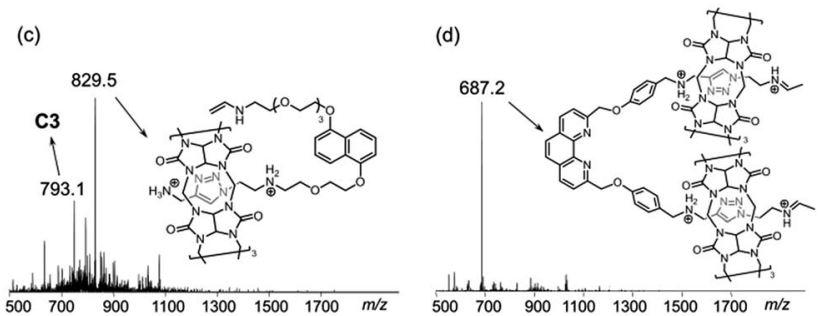

Fig. 1 (a) ESI-MS spectrum of C6; (b) HRMS of the peak at $m / z=915.5$ (left: experimental; right: simulation); (c) the $\mathrm{MS}^{2}$ and (d) $\mathrm{MS}^{3}$ spectra of c6.

COSY, NOESY and DOSY). The ${ }^{1} \mathrm{H}$ spectrum (400 MHz, $\mathrm{D}_{2} \mathrm{O}$ and 298 K) of C6 shows one set of resonances, indicating a highly symmetrical structure of $\mathbf{C 6}$ in aqueous solution. While the ${ }^{1} \mathrm{H}$ resonances from the $\mathrm{CB}[6]$ in $\mathbf{C 6}$ may be obscured by the resonances of other aliphatic protons in the molecule and the four inequivalent chemical environments of the $\mathrm{CB}[6]$ methylene protons can only be vaguely observed in the ${ }^{1} \mathrm{H}$ spectrum, the ${ }^{13} \mathrm{C}\left\{{ }^{1} \mathrm{H}\right\}$ spectrum of $\mathbf{C 6}$ clearly shows the two different chemical environments of the carbonyl (at 156.3 and $156.5 \mathrm{ppm}$ ) and methylene (at 51.4 and $51.7 \mathrm{ppm}$ ) carbons, which are the result of the interlocking of $\mathrm{CB}[6]$ on the unsymmetrical triazole (Fig. S34 $\dagger$ ). In addition, all the ${ }^{13} \mathrm{C}$ resonances of the $\mathrm{CB}[6]$ carbons (156.5, 156.3, 70.4, 51.7 and 51.4 ppm) in C6 are upfield shifted by ca. $4 \mathrm{ppm}$ when compared to that of the guest-free $\mathrm{CB}$ [6] (160.0, 74.2 and $55.3 \mathrm{ppm}){ }^{23}$ further confirming the interlocking of the macrocycle on the [6]catenane. Comparing the ${ }^{1} \mathrm{H}$ spectra of $\mathbf{C 6}$ and $\mathbf{C 3}$, the phenylene protons of $\mathbf{C 6}$ are upfield shifted by 0.59 and $1.14 \mathrm{ppm}$, while the phenanthroline protons are downfield shifted by $0.26,0.24$ and 0.29 ppm, suggesting close proximity of the phenylene and phenanthroline units with an edge to face orientation (Fig. 2). These chemical shift changes are comparable to those observed between the phenanthroline ligand $\mathbf{1}$ and the $\mathrm{Cu}^{\mathrm{I}}$ complex $\left[\mathrm{Cu}(\mathbf{1})_{2}\right]\left[\left(\mathrm{PF}_{6}\right)\right]$ (Fig. S37†), showing the presence of the same $\mathrm{Cu}^{\mathrm{I}}$-phenanthroline coordination motif in C6. On the other hand, the triazole protons of $\mathbf{C 6}$ and $\mathbf{C} 3$ at 6.31 and 6.23 ppm, respectively, are significantly upfield shifted when compared to that of the reference compound which lacks the $\mathrm{CB}[6]$ binding at $7.72 \mathrm{ppm}$

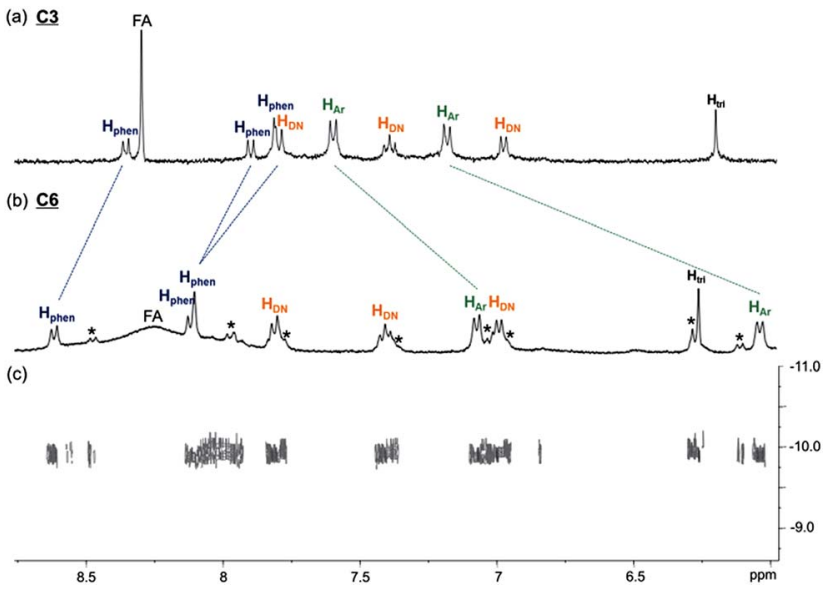

Fig. 2 Partial ${ }^{1} \mathrm{H}$ NMR (400 MHz, $\mathrm{D}_{2} \mathrm{O}$ and $298 \mathrm{~K}$ ) of (a) $\mathrm{C} 3$ and (b) C6. ${ }^{1} \mathrm{H}$ resonances from the phenanthroline, naphthalene, phenylene and triazole units are labelled with $\mathrm{H}_{\text {phen, }} \mathrm{H}_{\mathrm{DN}}, \mathrm{H}_{\text {Ar }}$ and $\mathrm{H}_{\text {tri }}$ respectively. Resonances from the copper-free $\mathrm{C} 6$ are labelled with *. Signals at $\mathrm{ca}$. $8.3 \mathrm{ppm}$ are assigned as the residual formate (FA) from preparative HPLC (see the ESI† for details); (c) partial 2D DOSY (500 MHz, $\mathrm{D}_{2} \mathrm{O}$ and 298 K) of C6.

(Fig. S20 $\dagger$ ), consistent with the inclusion of the triazole in the cavity of $\mathrm{CB}[6]$ in both $\mathbf{C 6}$ and $\mathbf{C 3} .^{18}$ The close proximity between the phenylene and phenanthroline units in C6, and that between the triazole and $\mathrm{CB}[6]$ is also supported by the corresponding NOE cross peaks in the 2D NOESY spectrum (Fig. S36 $\dagger^{\dagger}$. Careful analysis of the ${ }^{1} \mathrm{H}$ spectrum of $\mathbf{C 6}$ revealed the presence of a minor species. Further LCMS analysis of the isolated C6 sample from preparative HPLC showed a minor

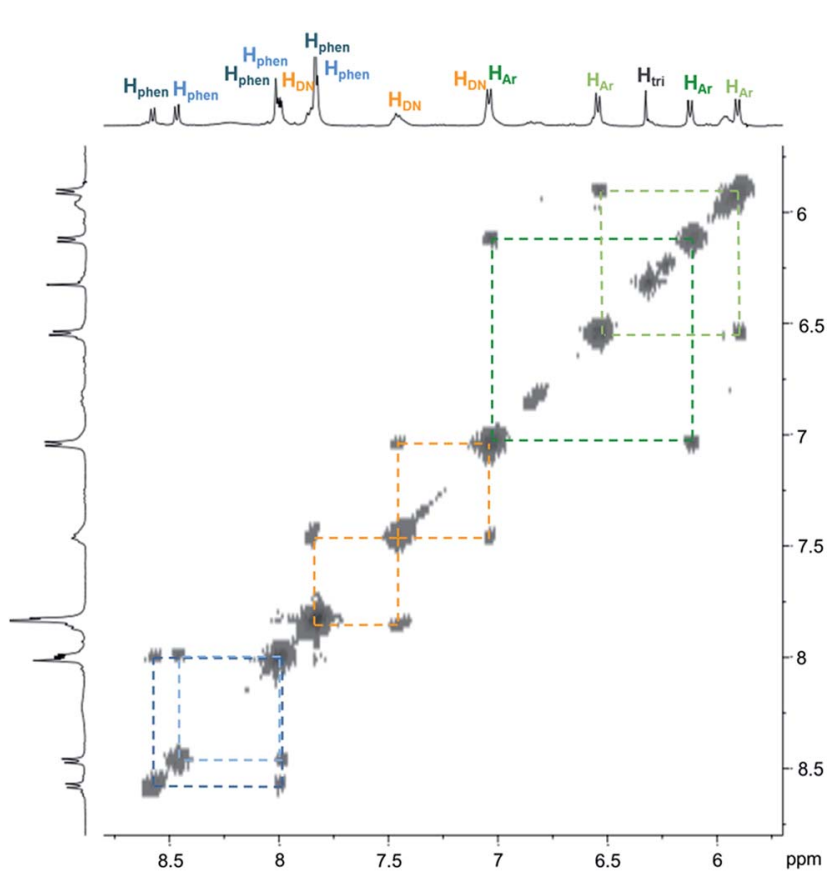

Fig. 3 Partial COSY (500 MHz, $\mathrm{D}_{2} \mathrm{O}$ and $298 \mathrm{~K}$ ) of C4. Correlations within the two inequivalent phenanthrolines and phenylenes are highlighted by dash lines. 
portion of the $\mathrm{Cu}^{+}$in $\mathbf{C 6}$ was decomplexed from the [6]catenane, suggesting that the set of minor resonances is due to the copper-free C6 (Fig. S5a $\dagger$ ). Diffusion ordered spectroscopy (DOSY) experiments showed that both sets of resonances have the same diffusion coefficient $(\log D=-9.93)$, further supporting the assignment of the minor component to the metal free form of the [6] catenane, as both the $\mathrm{Cu}^{+}$-complexed and $\mathrm{Cu}^{+}$-free forms of $\mathbf{C 6}$ are expected to have similar sizes and hydrodynamic volumes (Fig. 2c). Attempts to obtain a pure sample of the copper-free $\mathbf{C 6}$ through treating the $\mathrm{Cu}^{+}$-containing $\mathbf{C 6}$ with common demetallation reagents $\left(e . g . \mathrm{CN}^{-}\right)$were unsuccessful. The required alkaline medium is incompatible with the solubility of $\mathbf{C 6}$ so that the latter precipitated from the aqueous solution and the demetallation reaction could not be performed. $^{24}$

The formation of topological isomers, including those with different numbers of interlocking macrocycles or different interlocking patterns, connectivities or topologies, other than the target $[n]$ catenane that leads to low yield and difficult purification processes is one main reason for the low efficiency of $[n]$ catenane assembly. Notably, the use of $\mathrm{CB}[6]$-catalysed azide-alkyne cycloaddition to ring-close the precursors in our strategy not only resulted in a good yield of $\mathbf{C 6}$ due to its high efficiency in bond formation and ring-closure, but the prerequisite $\mathrm{CB}[6]$ binding for ring-closure also simultaneously ensures that the macrocycle will be interlocked and therefore minimises the formation of other catenanes with less interlocked macrocycles. While the $\mathrm{CB}[6]$-catalysed reaction has been employed to obtain rotaxanes, its application for cyclising and synthesising catenanes has not yet been demonstrated as far as we are concerned. ${ }^{18}$ In addition, the use of different orthogonal interactions as templates also directs the macrocycles to their designated interlocking site with good fidelity. The formation of [6]catenanes other than C6 with different topologies or connectivity patterns is minimised. Only the branched structure with one cross-point for each interlocking pair of the macrocycles was identified. Moreover, the flexibility of the diazide 2 , in conjunction with the dilute conditions used for the macrocyclisation reaction, also favours only the $[1+1]$ cyclisation that leads to $\mathbf{C 6}$ but not other higher order $[n]$ catenanes with larger macrocycles derived from other cyclic oligomers (Fig. S3†). Taken together, all of these effects help to suppress the formation of undesired topological isomers other than $\mathbf{C 6}$ and highlight the effectiveness of our strategy in its synthesis.

The presence of the $\pi$ rich dioxynaphthalene units in C6 (and C3) offers an opportunity to further interlock a $\pi$ deficient macrocycle to give a higher order $[n]$ catenane. Introduction of the $\pi$-deficient cyclobisparaquat $\left(p\right.$-phenylene) $\left(\mathrm{CBPQT}^{4+}\right)$ macrocycle to the $\mathrm{CB}[6]$-catalysed click reaction between $\mathbf{1}$ and 2 , however, did not result in any higher order $[n]$ catenane, only $\mathbf{C 3}$ and the free $\mathrm{CBPQT}^{4+}$ macrocycle (Fig. S4 $\dagger$ ). The failure to incorporate $\mathrm{CBPQT}^{4+}$ into any cyclised product is probably due to the repulsive Columbic interaction between $\mathrm{CBPQT}^{4+}$ and 2 under the acidic reaction conditions which prohibits the formation of the charge transfer complex (see the ESI $\dagger$ for details). On the other hand, the compatibility of the $\mathrm{Cu}^{\mathrm{I}}$-phenanthroline coordination and $\mathrm{CB}[6]$-ammonium binding as demonstrated by the successful synthesis of $\mathbf{C 6}$ prompted us to study and diversify the use of other phenanthroline building blocks to synthesise other interlocked structures. Following a similar procedure, a $\mathrm{CB}[6]$-catalysed click reaction between 2 and the heteroleptic complex $[\mathrm{Cu}(\mathbf{1})(3)]\left[\left(\mathrm{PF}_{6}\right)\right]$ afforded the $[4]$ catenane $\mathbf{C 4}$ in $84 \%$ yield as demonstrated through LCMS analysis of the crude product mixture (Scheme 3). Data from MS, MS $^{n}$ and NMR studies (Fig. 3, S28-32 and S40†) on C4 are all consistent with the expected [4]catenane structure with three different types of macrocycles interlocked in a radial fashion. The two inequivalent chemical environments for the phenanthroline and phenylene units due to the unsymmetrical coordination environment can be clearly observed and identified from the 2D COSY spectrum (Fig. 3). [n]Catenanes with three or more types of macrocycle are not common. ${ }^{5}$ Correctly positioning the different macrocycles through using orthogonal templates is one of the keys to the successful synthesis of the [4] catenane, otherwise other topological and/or positional isomers will result. Incorporation of different types of macrocycle into a single $[n]$ catenane will facilitate further functionalisation with

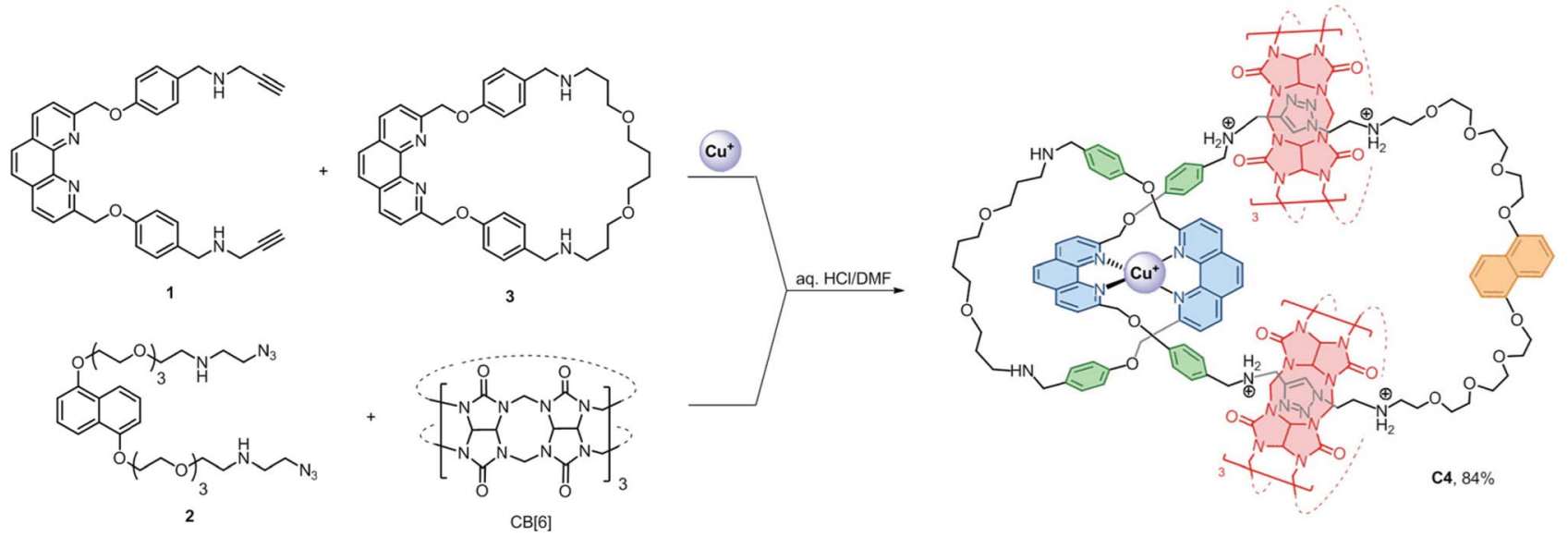

Scheme 3 Synthesis of C4. Its structure is shown as the +5 ion which is the most abundant and stable form as observed in the ESI-MS study. 
good selectivity and/or integration of the interlocked compound into different materials. The successful syntheses of $\mathbf{C 4}$ and $\mathbf{C 6}$ demonstrate that the present strategy is effective and modular. By proper design of the precursor building blocks and control of the reaction conditions, two different high order $[n]$ catenanes (C4 and C6) can be facilely and selectively obtained. The strategy is controllable and could be easily extended to other interlocked structures with different numbers of interlocking macrocycles and connectivity.

\section{Conclusions}

In summary, a new strategy that employs orthogonal supramolecular interactions (metal-ligand coordination, ion-dipole and hydrophobic interactions) and an efficient bond forming reaction ( $\mathrm{CB}[6]$-catalysed click) to preorganise and construct multi-macrocyclic $[n]$ catenanes has been successfully demonstrated through the efficient syntheses of the [3]-, [4]- and [6] catenane products, C3, C4 and C6. Their syntheses are highly facile and the yields are good ( $>80 \%)$. This modular approach to $[n]$ catenane will serve not only as a starting point to a general synthetic method that can be extended to higher order $[n]$ catenanes with more interlocked macrocycles and different topological patterns of the interlocking macrocycles, but the new interlocked molecules could also be novel candidates for the development of new functional molecular materials that are based on the unique mechanical properties and structural complexities of the $[n]$ catenanes. Further studies to extend our strategy to other high order $[n]$ catenanes with more interlocked rings and/or different interlocking topologies and connectivity patterns in a predictable and controllable way, and subsequent studies on their molecular motions are currently under way.

\section{Acknowledgements}

The work described in this paper was supported by a grant from the Research Grants Council of the Hong Kong Special Administration Region, China (Early Career Scheme, Project No. HKU 27300014) and the Croucher Foundation. KW and CCY acknowledge the receipt of the Postgraduate Scholarship from The University of Hong Kong. We also thank Dr Eva Y. M. Fung and Prof. C. M. Che for their help in the HRMS experiments, and Ms Bonnie Yan for her technical assistance in the NMR experiments.

\section{Notes and references}

1 (a) G. Gil-Ramírez, D. A. Leigh and A. J. Stephens, Angew. Chem., Int. Ed., 2015, 54, 6110-6150; (b) N. H. Evans and P. D. Beer, Chem. Soc. Rev., 2014, 43, 4658-4683; (c) J. E. Beves, B. A. Blight, C. J. Campbell, D. A. Leigh and R. T. McBurney, Angew. Chem., Int. Ed., 2011, 50, 92609327; (d) L. Fang, M. A. Olson, D. Benítez, E. Tkatchouk, W. A. Goddard and J. F. Stoddart, Chem. Soc. Rev., 2010, 39, 17-29; (e) K. D. Hännia and D. A. Leigh, Chem. Soc. Rev., 2010, 39, 1240-1251; (f) Z. Niu and H. W. Gibson, Chem. Rev., 2009, 109, 6024-6046.
2 (a) Y. Ye, S.-P. Wang, B. Zhu, T. R. Cook, J. Wu, S. Li and P. J. Stang, Org. Lett., 2015, 17, 2804-2807; (b) S. Li, J. Huang, T. R. Cook, J. B. Pollock, H. Kim, K.-W. Chi and P. J. Stang, J. Am. Chem. Soc., 2013, 135, 2084-2087; (c) S. Li, M. Liu, B. Zheng, K. Zhu, F. Wang, N. Li, X.-L. Zhao and F. Huang, Org. Lett., 2009, 11, 3350-3353.

3 (a) J. E. Beves, J. J. Danon, D. A. Leigh, J.-F. Lemonnier and I. J. Vitorica-Yrezabal, Angew. Chem., Int. Ed., 2015, 54, 7555-7559; (b) C. Schouwey, J. J. Holstein, R. Scopelliti, K. O. Zhurov, K. O. Nagornov, Y. O. Tsybin, O. S. Smart, G. Bricogne and K. Severin, Angew. Chem., Int. Ed., 2014, 53, 11261-11265; (c) J.-F. Ayme, J. E. Beves, C. J. Campbell and D. A. Leigh, Angew. Chem., Int. Ed., 2014, 53, 78237827; (d) N. Ponnuswamy, F. B. L. Cougnon, G. D. Pantoș and J. K. M. Sanders, J. Am. Chem. Soc., 2014, 136, 8243-8251. 4 D. A. Leigh, R. G. Pritchard and A. J. Stephens, Nat. Chem., 2014, 6, 978-982.

5 D. B. Amabilino, P. R. Ashton, V. Balzani, S. E. Boyd, A. Credi, J. Y. Lee, S. Menzer, J. F. Stoddart, M. Venturi and D. J. Williams, J. Am. Chem. Soc., 1998, 120, 4295-4307.

6 S. P. Black, A. R. Stefankiewicz, M. M. J. Smulders, D. Sattler, C. A. Schalley, J. R. Nitschke and J. K. M. Sanders, Angew. Chem., Int. Ed., 2013, 52, 5749-5752.

7 M. J. Langton, J. D. Matichak, A. L. Thompson and H. L. Anderson, Chem. Sci., 2011, 2, 1897-1901.

8 S. Li, J. Huang, F. Zhou, T. R. Cook, X. Yan, Y. Ye, B. Zhu, B. Zheng and P. J. Stang, J. Am. Chem. Soc., 2014, 136, 5908-5911.

9 C.-F. Chang, C.-J. Chuang, C.-C. Lai, Y.-H. Liu, S.-M. Peng and S. H. Chiu, Angew. Chem., Int. Ed., 2012, 51, 1009410098.

10 S. Dasgupta and J. Wu, Org. Biomol. Chem., 2011, 9, 35043515.

11 K.-M. Park, S.-Y. Kim, J. Heo, D. Whang, S. Sakamoto, K. Yamaguchi and K. Kim, J. Am. Chem. Soc., 2002, 124, 2140-2147.

12 S.-G. Roh, K.-M. Park, G.-J. Park, S. Sakamoto, K. Yamaguchi and K. Kim, Angew. Chem., Int. Ed., 1999, 38, 637-641.

13 F. Bitsch, C. O. Dietrich-Buchecker, A. K. Khémiss, J.-P. Sauvage and A. Vandorsselaer, J. Am. Chem. Soc., 1991, 113, 4023-4025.

14 H. Iwamoto, S. Tafuku, Y. Sato, W. Takizawa, W. Katagiri, E. Tayama, E. Hasegawa, Y. Fukazawab and T. Haino, Chem. Commun., 2016, 52, 319-322.

15 (a) P. Wei, X. Yan and F. Huang, Chem. Soc. Rev., 2015, 44, 815-832; (b) X.-Y. Hu, T. Xiao, C. Lin, F. Huang and L. Wang, Acc. Chem. Res., 2014, 47, 2041-2051; (c) M. L. Saha, S. De, S. Pramanik and M. Schmittel, Chem. Soc. Rev., 2013, 42, 6860-6909; (d) C.-H. Wong and S. C. Zimmerman, Chem. Commun., 2013, 49, 1679-1695.

16 (a) J.-P. Sauvage, Acc. Chem. Res., 1990, 23, 319-327; (b) C. O. Dietrich-Buchecker and J.-P. Sauvage, Chem. Rev., 1987, 87, 795-810.

17 (a) W. L. Mock and N.-Y. Shih, J. Org. Chem., 1983, 48, 36193620; (b) W. L. Mock, T. A. Irra, J. P. Wepsiec and M. Adhya, J. Org. Chem., 1989, 54, 5302-5308. 
18 (a) M. K. Sinha, O. Reany, M. Yefet, M. Botoshansk and E. Keinan, Chem.-Eur. J., 2012, 18, 5589-5605; (b) G. Celtek, M. Artar, O. A. Scherman and D. Tuncel, Chem.Eur. J., 2009, 15, 10360-10363; (c) D. Tuncel and M. Katterle, Chem.-Eur. J., 2008, 14, 4110-4116; (d) D. Tuncel, Ö. Özsar, H. B. Tiftika and B. Salih, Chem. Commun., 2007, 1369-1371; (e) D. Tuncel and J. H. G. Steinke, Macromolecules, 2004, 37, 288-302; $(f)$ K. Kim, Chem. Soc. Rev., 2002, 31, 96-107; (g) D. Tuncel and J. H. G. Steinke, Chem. Commun., 1999, 1509-1510.

19 Yields of C3, C4 and C6 syntheses were calculated based on the relative peak areas in the HPLC chromatograms. Relative absorbances of different chromophores were determined using independent UV-Vis measurements. The $[n]$ catenanes were also isolated from preparative HPLC and the isolated yields of $\mathbf{C 3}, \mathbf{C 4}$ and $\mathbf{C 6}$ were determined to be $66 \%, 54 \%$, and $69 \%$ respectively. See $\mathrm{ESI} \dagger$ for details.

20 (a) F. B. L. Cougnon, N. Ponnuswamy, G. D. Pantoş and J. K. M. Sanders, Org. Biomol. Chem., 2015, 13, 2927-2930; (b) M. J. Langton and P. D. Beer, Chem. Commun., 2014, 50, 8124-8127; (c) S. Grunder, P. L. McGrier, A. C. Whalley, M. M. Boyle, C. Stern and J. F. Stoddart, J. Am. Chem. Soc.,
2013, 135, 17691-17694; (d) R. S. Forgan, J. J. Gassensmith, D. B. Cordes, M. M. Boyle, K. J. Hartlieb, D. C. Friedman, A. M. Z. Slawin and J. F. Stoddart, J. Am. Chem. Soc., 2012, 134, 17007-17010.

21 D. Whang, K.-M. Park, J. Heo and K. Kim, J. Am. Chem. Soc., 1998, 120, 4899-4900.

22 (a) S. W. Heo, T. S. Choi, K. M. Park, Y. H. Ko, S. B. Kim, K. Kim and H. I. Kim, Anal. Chem., 2011, 83, 7916-7923; (b) D. V. Dearden, T. A. Ferrell, M. C. Asplund, L. W. Zilch, R. R. Julian and M. F. Jarrold, J. Phys. Chem. A, 2009, 113, 989-996; (c) H. Zhang, E. S. Paulsen, K. A. Walker, K. E. Krakowia and D. V. Dearden, J. Am. Chem. Soc., 2003, 125, 9284-9285.

23 J. Kim, I.-S. Jung, S.-Y. Kim, E. Lee, J.-K. Kang, S. Sakamoto, K. Yamaguchi and K. Kim, J. Am. Chem. Soc., 2000, 122, 540541.

24 Preliminary LCMS analysis of a purified aqueous sample of C6 after prolonged standing at $4{ }^{\circ} \mathrm{C}$ for 4 months could give the copper free, demetalated [6]catenane in about $50 \%$ yield (Fig. S5 $\dagger$ ). Presumably, the $\mathrm{Cu}^{\mathrm{I}}$ was slowly oxidised under air to $\mathrm{Cu}^{\mathrm{II}}$, which has a weaker binding to the phenanthroline coordination pocket in the catenane. 